Report on Third International Workshop on Weak Interactions*

\section{K. Tanaka}

Department of Physics The Ohio, State University

Columbus, Ohio 43210
This report was prepoTICE

sponsored by the United Stotes Covernment. Nerther

the United States nor the United States Enerer

Research and Development Administration, noI any of

their employees, nor any of their contractors,
subcontractors, or their warranty. express or implied, or assumes any any

or usefuln responsibility for the accuracy, completenes

process disclosed, or represents, apparatus, product or

infringe privately owned rights.

Supported in part by U.S. Energy Research and Development Administration under Contract AT(11-1) 1545.

\title{
MASTER
}




\section{DISCLAIMER}

This report was prepared as an account of work sponsored by an agency of the United States Government. Neither the United States Government nor any agency Thereof, nor any of their employees, makes any warranty, express or implied, or assumes any legal liability or responsibility for the accuracy, completeness, or usefulness of any information, apparatus, product, or process disclosed, or represents that its use would not infringe privately owned rights. Reference herein to any specific commercial product, process, or service by trade name, trademark, manufacturer, or otherwise does not necessarily constitute or imply its endorsement, recommendation, or favoring by the United States Government or any agency thereof. The views and opinions of authors expressed herein do not necessarily state or reflect those of the United States Government or any agency thereof. 


\section{DISCLAIMER}

Portions of this document may be illegible in electronic image products. Images are produced from the best available original document. 


\section{INTRODUCTION}

The International Workshop on Weak Interactions

The Third International Workshop on Weak Interactions with Very High Energy beams was held at Columbus, Ohio during September $3-13$. The first Workshop was held at Skovde, Sweden on 1973 and the second Workshop held at Strob1, Austria in 1974. A fourth is scheduled at Trieste, Italy in 1976 and the fifth in Japan in 1977. The number of participants of the first two were around 50 and the third one had approximately 80 participants representing 10 countries with 24 speakers and 9 discussion leaders: It was of the optimum size that facilitated rapport between the speaker and the participants during the general lectures that were held in the mornings and early afternoons. This was followed by discussion groups in parallel sesșions. The Workshop is planned to be a meeting that is different from a large international conference and a summer school. Small groups of people of similar interest join together in discussion sessions and work out:new ideas or try to elucidate various questions that may come up. The human aspects are emphasized so that one can get to know each other and establish personal contacts.

With the support of the Chairman, Dr.E.L. Jossem, we obtained fiscal support from the following: College of Mathematics and Physical Sciences of Ohio State University, Graduate School of The Ohio State University, Ohio State Development Fund and U.S. Energy. Research and Development Administration. We regard the generous support that The Ohio State University rendered toward the Workshop a strong affirmation of the universities' commitment toward support of basic research. 
The members of the international advisory committe who rendered invaluable help in the program are: B.W. Lee, R.E. Marshak, Nambu, J. Nilsson, H. Pietschmann, and S.B. Treiman; members of the local committee who assisted in the arrangements of the Workshop are A Lesnik, B.G. Mainland, W.F. Palmer, S.S. Pinsky, N.W. Reáy, K. Reibel; T.A. Romanowski, D.M. Scott, N.R. Stanton, W.W. Wada and K. Tanaka (chair$\operatorname{man})$. 
II. PRंOGRAM

The main topics of the Workshop can be grouped into the following with their respective speakers:

1. $v$ Interaction

2. Charm

3. Gauge Models

4. Quark Models

5. CP Violation

6. Symmetry

7. Hyperon Beam Experiments
C. Rubbia, L. Wolfenstein, H. Faissner;

R. Palmer, H. Pietschmann, M. Derrick;

S. Adler, L. Stutte, W. Lee, R.N. Diamond,

P. Schreiner.

S.B. Treiman, N.W. Reay.

B.W. Lee, R.N. Mohapatra.

Y. Nambu, R. Oakes, C. Albright.

A. Pais, R.G. Sachs.

G. Marx, A. Pais, W. Kummer.

There was an excellent review of the "Photon Lepton Symposium". (Stanford USA)

by G. Kramer, and the "Fifth International Neutrino Conference" (Balaton, Hungary) during discussions.

The discussion sessions are the following with their respective leaders:

Topics on Hidden Quantum Numbers

G. Kramer

Gauge Theories

A. Zee

Neutra1 Currents

A. Paschos

Topics on Gauge Models

M.K. Gaillard

Topics on Heavy Leptons

C. Jarlskog

Models of CP Violation

B. Svensson

Weak Interactions at SuperHigh Energies

J.R. Primack 
Infrared Behavior of ymptotic Free Theories

Hyperon Beams

ropics on Supersyminetry
R.A. Brandt

M.K. Gaillard

H. Stremnitzer

The discussion leaders conducted such stimulating and int $1 \cdot \cdots \cdot \cdots$ ing sessions that many participants had difficulty deciding which sessimili to attend.

The following seminars were given:

Cabibbo Angle and

Quark Mass Spectrum

A. Zee

Second Class Currents

S. Nakamura

Non-Linear Field Theory

Aspects of Gauge Theory

D.Y. Kim

$\checkmark$ Charged Current and

Charn Production

'I' Weiler

socond class currents

R. T'eese

\section{II.I . LECTURES}

The speakers gave timely, comprehensive and excellent lectures that were appreciated by all the participants. In this section an attempt is inade to briefly summarize the talks, avoiding as much as possible detailed scientific information that is available in the literature. The author hopes he has done justice to the talks and takes responsibility for any. arrors that may have been introduced.

The new experimental data is presented first and then the theoretj.cal discussions,

1. Dimuon Events (Rubhia) - Dimuon events on $\nu$ interactions has been produced by three different beams $\left(\nu_{\mu}\right.$ horn focusing, $\nu_{\mu} \bar{\nu}_{\mu}$ quadrupole triplet focusing and $\bar{v}_{\mu}$ horn focusing). Harvard-Wisconsin-PennsylvaniaFermilab group has seen the following dimuon events,

$$
\begin{aligned}
& \nu_{\mu} L^{2} \rightarrow \mu^{+} \mu-\nu_{\mu} \times, \quad \mu^{-} \mu^{-} \bar{\nu}_{\mu} \times \\
& V_{\mu} N \rightarrow \mu^{+} \mu-\bar{\nu}_{\mu} \times, \quad \mu^{+} \mu^{-} \nu_{\mu} \times \text { (outside fiducial volume) }
\end{aligned}
$$


and have not seen the trimuon events

$$
\nu_{\mu} N \rightarrow \mu^{-} \mu^{+} \mu{ }^{-} X \text { and } \bar{\nu}_{\mu i} \mathcal{N}^{-} \mu^{+} \mu^{+} \mu^{-} X \text {. }
$$

The ratio of the rates are

$$
\begin{aligned}
& \frac{R\left(\mu^{+} \mu^{-} \nu x\right)}{R\left(\mu^{-} x\right)}=(0.8 \pm 0.2) 10^{-2} \\
& \frac{R\left(\mu^{-} \mu^{-} \nu x\right)}{R\left(\mu^{-} \mu^{+} \nu x\right)}=0.1 \pm 0.05 \\
& \frac{R\left(\mu^{+} \mu^{-} \nu x\right)}{R\left(\mu^{+} x\right)}=2 \times 10^{-2} \text { (bosed on } 4 \text { events). }
\end{aligned}
$$

The ratio of average laboratory energy of $\mu^{-}$and $\mu^{+}$is

$$
\langle E(\mu-j) /\langle E(\mu)\rangle=3.7 \pm 0.6 .5
$$

and $P_{\mu^{-}}>P_{\mu}$ for $\nu$ beam and $P_{\mu^{+}}>P_{\mu}-$ for $\bar{\nu}$ beam.

2. One $\pi^{0}$ Production in Neutrino Interaction (Faissner) - The ratio of the neutral current events (NC) with charge current events (CC) for $\nu$ interaction 18 (Gargamelle)

$$
R_{n}^{\prime}=N C / C
$$

$$
0,35 \leq R_{0}^{i} \leq 0,40 \pm 0.05
$$

$\bar{\nu}$ interaction is

$$
\bar{R}_{0}^{\prime}=0.55 \pm 0.07
$$

where the final states of $\mathrm{NC} \cdot$ is $\mathrm{m}$ proton + one or two $Y$ or no proton and two $\mathcal{C}$ $\mathrm{CC}$ is $1 \mu+\mathrm{m}$ proton + one or two $\gamma$. 
3. Single Pion Production in Neutrino Interactions (Lee) - The ratio of NC with CC for single $\pi^{\circ}$ production in $\nu$ interaction at Brookhaven Lab. is

$$
R^{\prime}=0.17 \pm 0.04
$$

where

$$
\begin{array}{cc}
\mathrm{CC} \text { is } 1 \mu+1 \pi^{0}(\eta)+n p \\
\mathrm{NC} \text { is } \quad 1 \pi^{0}(\eta)+n p
\end{array} \quad n=0,1
$$

The $R_{0}^{\prime}$ is to be compared with $R^{\prime}{ }_{0}$ (theory) $=0.19, \sin ^{2} \theta_{w}=0.39$ by Adler and there is no clear $\Delta$ production in the $N C$.

4. Double and Triple Pion Production in $v$-d Collisions (Derrick) - The ratio of single pion production at Argonne Lab. from $\mathrm{p}$ and $\mathrm{n}$ is $\Delta\left(\nu p \rightarrow \mu^{-} p \pi^{+}\right): A\left(\nu n \rightarrow \mu^{-} n \pi^{+}\right): A\left(\nu n \rightarrow \mu^{-} p \pi^{0}\right)=119: 41: 56$ in contrast to $\Delta$ dominance $9: 1: 2$, so there is a resonant $T=\frac{3}{2}$ amplitude and a strong non resonant $T=\frac{1}{2}$ amplitude. The cross sections of $n$ pion production $\sigma_{n \pi}$ all equal to $10^{-38} \mathrm{~cm}$ at $\mathrm{E}=6 \mathrm{GeV}$ where $1 \pi, 2 \pi$, and $3 \pi$ are respectively $\mu^{-} p \pi^{+}, \mu^{-} p \pi^{+} \pi^{-}$and $\mu^{-} p \pi^{+} \pi^{+} \pi^{-}$. Mean multiplicities $\langle\mathrm{n}\rangle$ at $\mathrm{E}=6 \mathrm{GeV}$ are $2.30 \pm 0.10$ for $v p$ and $1.45 \pm 0.12$ for $v n$. The mean charged hadron multiplicity is given by

$$
<n_{c}>=1.09 \pm 0.87+1.13 \ln S \text {. }
$$

5. Neutrino Interactions on Protons in the Fermi Lab 15-ft Bubble Chamber (Diamond) - Mean charged hadron multiplicity is

$$
\therefore \quad\left\langle n_{c}\right\rangle=1.09 \pm 0.7+(1.13 \pm 0.12) \ln w^{2}
$$

In deep inelastic distributions there is a slight excess at low $\mathrm{x}$ and the $y$ distribution is consistent with a flat distribution. The ratio of $\Delta S=-\Delta Q$ to strange decays is $1 / 30$ compred to BNL $1 / 5$ (Palmer). 
6. $\overline{v p}$ Interaction (Schreiner) - The average multiplicites for $E_{\vec{v}} ? 30 \mathrm{GeV}$ are $\langle n\rangle=2.66 \pm 0.16,\left\langle\pi^{\circ}\right\rangle=2.11 \pm 0.52,\langle\Lambda\rangle=0.14 \pm 0.06$ and $\left\langle\mathrm{K}^{\circ}\right\rangle$ $0.15 \pm 0.08$ and no charm peaks are found. The $\mathbb{X}, y, Q^{2}, W,\langle\pi>$ agree with the parton model and strange particle productions are not abnormally large. These are our first result in the 15-ft chamber at Fermilab.

7. Recent results from Cal-Tech Fermi Lab. Experiment - (Stutte) The latest value of

$$
R=\left(\frac{N C}{C C}\right)_{V}=0.21 \quad \bar{R}=\left(\frac{N C}{C C}\right)_{\bar{V}}=0.43
$$

and V-A theory look good except for renormalization.

The dimuon events

$\begin{array}{ccc} & v & \bar{\nu} \\ 1 \mu & 2313 & 446 \\ 2 \mu & 19 & 2\end{array}$.

The results of fit for CC $x=Q^{2} / 2 M \nu\left\langle 0.1, y=\nu / E_{\nu}\right.$ are

$$
\begin{aligned}
& d \sigma^{2} / d y \sim 1+0.3(1-y)^{2} \\
& d \sigma^{\bar{\nu}} / d y \sim 0.3+(1-y)^{2} .
\end{aligned}
$$

8. Neutrino Results from BNL 7-ft Bubble Chamber (Palmer) - The crosssection ratio is

$$
6(\nu n) / 6(\nu p)=1.4 \pm 0.14
$$

where the theoretical predictions for this ratio are $1.96,1.59$, and 1.58. There is a $\Delta S=-\Delta Q$ strange particle production event

$$
\nu p \rightarrow \mu-\pi^{+} \pi^{+} \pi^{+} \pi^{-} \wedge
$$

at $\mathrm{E}_{\nu}=13.5 \mathrm{GeV}$.

The ratio of $\Delta S=-\Delta Q$ to strange decays is $1 / 5$ and to non strange. decays is $0.7 \%$.

The ratio $\mathrm{NC} / \mathrm{CC}$ for $\mathrm{p}$ and $\mathrm{n}$ are $\quad \mathrm{NC} / \mathrm{CC}=0.28 \pm 0.1, \mathrm{p}$

$$
\mathrm{NC} / \mathrm{CC}=0.15+0.07 \cdot \mathrm{n}
$$


9. Leptonic Weak Neutral Currents (Faissner) - Gargamelle found $3 \pm 0.15$ clean events in $\bar{v}_{\mu}$ e scattering. The Aachen Padua group found candidates for $\nu_{\mu}$ e. The previous scattering events are reviewed and the possible background are carefully discussed.

10. Search for Charmed Particles (Reay) - At Fermilab experiments neutrino were incident on $\mathrm{B}_{e}$ target to search for the decay modes of charmed particles. The channels $\mathrm{K}^{-} \pi^{+}, \pi^{+} \pi^{-}, \mathrm{K}^{-} \mathrm{P}$ and $\overline{\mathrm{p}} \mathrm{p}$ yielded no charmed particles. Four possible reasons that nothing, was found are, the experiment is insensitive, the two body decay modes are not dominant, charmed particles live too long, and finally there is no charmed particle.

11. Experiments with High Energy Neutral and Charged Hyperon Beams at CERN (Overseth and Gaillard) Some results on total cross sections are

$$
\begin{array}{ll}
\sigma(\wedge p)=34.6 \pm 0.4 \mathrm{mb} & \sigma(\wedge n)=74 \pm 0.8 \mathrm{mb} \\
\sigma(\bar{n} p)=56 \pm 11 \mathrm{mb} & \sigma(\pi n)=46 \pm 20 \mathrm{mb}
\end{array}
$$

at $4.15 \mathrm{GeV} / \mathrm{c}$.

The limits on $\Delta S=2$ decay is

$\left(\Xi^{\circ} \rightarrow p \pi^{-}\right) /\left(\Xi^{0} \rightarrow \wedge \pi^{0}\right)<3.6 \times 10^{-5}$.

Preliminary measurement of the life time of $\sum^{0}$ is

$$
\tau\left(\Sigma^{\circ}\right)=(0.63 \pm 0.30) \times 10^{-19} \mathrm{sec}
$$


Further

$$
\begin{aligned}
& B R\left(\Xi^{0} \rightarrow \wedge \gamma\right)=(1.4 \pm 0.4) \times 10^{-3} \quad \alpha\left(\Xi^{0} \rightarrow \wedge \gamma\right)=-0.22 \pm 0.34 \\
& B R\left(\Xi^{0} \rightarrow \Sigma^{0} \gamma\right)=(2.2 \pm 1.2) \times 10^{-3} \\
& B R\left[\left(K_{s}^{0} \rightarrow \pi^{+} \pi^{-} \gamma\right) /\left(K_{s}^{0} \rightarrow \pi^{+} \pi^{0}\right)\right]=2.59 \times 10^{-3} .
\end{aligned}
$$

Future possibilities are $\Delta S=2$ semileptonic decays, form factors of $\Lambda^{\circ}$, parity violation in $\Sigma^{0} \rightarrow \wedge \gamma, \pi^{0} \rightarrow e^{+} e^{-}$decay, search for $T$ violation in $\Xi^{0} \Xi^{-}$decays $\mathrm{CP}$ studies in $\Xi^{0} \rightarrow \wedge \pi^{0}$ and $\bar{\Xi}^{0} \rightarrow \bar{\Lambda}^{\circ}$.

Treiman emphasized the charm picture as a possible interpretation. of the recent data on dimuon events. In $v$ induced reactions a pair of $\mu^{+}$ and $\mu^{-}$muons, dimuons is observed in the final state. The muon energy spectra rule out the process $\nu N \rightarrow L^{0} \ldots, \quad, L^{0} \rightarrow \mu^{+} \mu^{-} \nu$.

The theoretical bounds on the ratio of the average laboratory energies for $\mu^{-}$and $\mu^{+}$(Pais and Treiman) is

$$
0.48\left\langle\left\langle E\left(\mu^{-}\right)\right\rangle ;\left\langle E\left(\mu^{+}\right)\right\rangle\langle 2,1\right.
$$

whereas data (Rubbia) is $3.7 \pm 0.65$, so $\mathrm{L}^{\circ}$ cannot account for the data. Thịs strengthens the picture $\nu N \rightarrow \mu^{-}+$charm + oridnary hadrons

$$
\text { charm } \rightarrow \mu^{+}+\nu+\text { hadron. }
$$

In weak interaction model, if the charged current $C C$ has $\Delta C \neq 0$ and if the sea is allowed, $\overline{v N} \rightarrow$ charm is suppressed because only the sea contributes, 
10

and $V N \rightarrow$ charm has a valence contribution that is suppressed by $\sin ^{2} \theta$ and a sea contribution,

so although both charm productions are suppressed, we expect

$$
\frac{\sigma^{\nu}(\Delta c=1)}{\sigma^{2}(\Delta c=-1)} \geq 1
$$

and

$$
\begin{aligned}
& \frac{\sigma^{\nu}(\Delta c=1)}{\sigma^{\nu}(\Delta c=0)}<0.1 \\
& \frac{\sigma^{\nu}\left(\mu^{+} \mu\right)}{\sigma^{\nu}\left(\mu^{-}\right)}<0,1 B
\end{aligned}
$$

where $B$ is the branching ratio for charm $\rightarrow \mu-\mu_{-}:$

Experimental value of $G^{\nu}\left(\mu^{+} \mu^{-}\right) / 6^{\nu}\left(\mu^{-}\right) \approx 0,0.1$ yields $B$. 20.1 In standard model $R\left(\mu^{-} \mu^{-} \nu x\right) / R\left(\mu^{-} \mu^{+} \nu x\right) \leq 10^{-3}$ compared to experimental ratio of 0.1 . To enhance charm production in $\nu$ and $\bar{\nu}$ interactions, models with more quarks than standard model and also right handed couplings are considered..

Wolfenstein reviewed the general form of neutral current phenomenology and stated the theorems. For pure $V$ or $A$ interaction $\sigma_{0}=6(\nu A \rightarrow \nu B)$ and $\bar{\sigma}_{0}=\sigma(\bar{\nu} A \rightarrow \bar{\nu} B)$ satisfy the following identity $\sigma_{0}=\bar{\sigma}_{0}$ from hermiticity Gargamelle experiment obtained $\quad \bar{\sigma}_{0}=(0.51 \pm 0.12) \sigma_{0}$

that must be compared with $\bar{\sigma}_{0}=0.9 .60$

where there is a $10 \%$ model dependent correction.

For pure $\Delta$ isovector production

$$
R_{0}^{\prime}=\sigma\left(\nu T \rightarrow \nu N^{\prime} \pi^{0}\right) / 2 \sigma\left(\nu T \rightarrow \mu N \pi^{0}\right)=\frac{1}{2}
$$

Experimental data

$$
\begin{array}{rlrl}
R_{0}^{\prime} & =0.17 \pm 0.04 & \text { BN } \\
& =0.15 \pm 0.05 \quad \text { Gargamelle }
\end{array}
$$


Single pion or $\Delta$ production on a isoscaler target yields information on the isotopic spin property of the NC

$$
\begin{aligned}
T=\frac{\left\langle\pi^{+}\right\rangle+\left\langle\pi^{-}\right\rangle-2\left\langle\pi^{0}\right\rangle}{\left\langle\pi^{+}\right\rangle+\left\langle\pi^{0}\right\rangle+\left\langle\pi^{-}\right\rangle} & =0 \text { for isocaler } \\
& =-1 \text { isovectior }
\end{aligned}
$$

where $\langle\pi\rangle$ is the average number of the respective pions. Tentative value from Gargamelle is $\mathrm{T}_{2}=-0.4$ so that $\mathrm{NC}$ has both isoscaler and isovector. Conclusion is that Weinberg Salam model with NC is OK with $\sin ^{2} \theta_{1,0} \approx 0,35$ and more tests in $\pi$ production, $\nu p \rightarrow \nu p$ $\nu(\bar{\nu})+e \rightarrow \nu(\bar{\nu})+e$, and $e^{+} e^{-} \rightarrow \mu^{+} \mu^{-} \quad$ are desirable.

Pietschmann reviewed the double differetial cross sections

$$
d^{2} \sigma^{N N} / d x d y \text { and } d^{2} \sigma^{\bar{N} N} / d x d y
$$

for charged and neutral currents.

When the valence quarks are considered and the sea contribution is neglected the $\mathrm{y}$ distribution for NC satisfy the equality (Ecker and Pietschmann).

$$
\frac{d \sigma^{L N}}{d y} / \sigma_{T}^{L N}=\frac{d \sigma^{T N}}{d y} / \sigma_{T}^{\overline{\nu N}}=1 \text { at } y=1-\frac{1}{\sqrt{3}}=0.42
$$
scattering and pion production by neutral current, and indicated that the ratio for low invariant mass $W<1.4 \mathrm{GeV}$ is

$$
\begin{aligned}
2 R_{0} & =\left[\sigma\left(\nu_{\mu} \rightarrow \nu_{\mu} n \pi^{0}\right)+\sigma\left(\nu_{\mu} p \rightarrow \nu_{\mu} p \pi^{0}\right)\right] / \sigma\left(\nu_{\mu} n \rightarrow \mu^{-} p \pi^{0}\right) \\
& =0.42 \quad\left(\sin ^{2} \theta_{\mu}=0.35\right) \\
& =0.51 \quad \text { (careful analysis) }
\end{aligned}
$$


This value corresponds to $R_{0}^{\prime}=0,15 \sim 0,18$ whereas

W. Lee reported $\cdots 0.17$ and Faissner. $0.35-0.40$.

If $\mathrm{P}_{33}$ is seen observed $R_{0}^{\prime}$ favors $\mathrm{SU}(2) \times \mathrm{x}(1)$ gauge-theory and if

$\mathrm{P}_{33}$ is not seen isoscalar neutral current structure is favored. .

There is a confusion theorem for NC that states that VA current and

SPT currents cannot be distinguished.

We next take up the topics, gauge models, quark models, CP.violation, and symmetry .

Lee reviewed nonleptonic decays, second order weak processes in minimal theory, and model building. The enhancement factor needed to explain the observed $\Delta I=\frac{1}{2}$ or octet rule in nonleptonic decays is around 2-3 in asymptotically free guage theories which is insufficient. The second order weak processes $K_{S} K_{L}$ mass differences and $K_{L}+\mu^{+} \mu^{-}$in minimal theory are consistent with the Glashow Iliopoulos Maiani model. In regard to model building a V+A term (DeRujula, George and Glashow) would explain nonleptonic $\Delta I=1 / 2$ rule, but there is trouble when $K \rightarrow .3 \pi$ and $K \rightarrow 2 \pi$ decays are related by current algebra methods (Holstein and Golowich) and for $K_{L}-K_{S}$ mass difference because of an additional diagram. The minimal theory with possible heavy leptons may survive for sometime.

Mohopatra introduced gauge models incorporating lepton quark symmetry ( 3 color quartets of quarks and 4 known leptons) and charm changing right handed currents and discussed $\Delta C=1$ nonleptonic decays, $\Delta S=2$ transitions and possible subquarks. The advantages of these gauge models the largest being $\mathrm{SU}(4) \mathrm{xSU}\left(4^{\prime}\right)$ are that the phenomenology, $\Delta I=\frac{1}{2}$ rule, $\mathrm{K}_{\mathrm{L}} \rightarrow 2 \pi$, and $\mathrm{CP}$ violation are incorporated. The new model can be tested in leptonic and hadronic NC that appèar in $\nu$ interactions. 
Nambu reviewed the general pattern and evolution of the quark model. It is further escalated into a 6 quark. model and has room for new quantum numpers, $\mathrm{CP}$ violation, and post quantum mechanics. (non-associative algebra). There are five exceptional Lie groups generated by octonions (Gursey et al). The subgroups of these groups lead to the natural interpretation of color and flavor of quarks. Quantum mechanical confinement combines ultraviolet asymptotic freedom and infrared slavery but where does the mass scale come from? Also classical confinement and various attempts at a non Abelian monopole theory are reviewed and the following questions are posed "Are both new quantum numbers and dynamics necessary?" "Is a new post quantum mechanics necessary?"

Albright and Oakes proposed a SU(4)/Z(2) sextet quark model in which the vector parts of the NC is pure isoscalar and the axial vector part is half isoscalar and half isovector. The predictions of the NC to CC ratio for $\nu$ and $\vec{\nu}$ scattering on isoscalar targets $N$ below charm threshold are $\hat{R}^{N}=0.25$ and $R^{\bar{N}}=0.44 ;$ also one finds

$$
\sigma(u+p \rightarrow L+p) / \sigma(\nu+n-\mu+p) \approx c_{1} 12
$$

The leptonic NC cross sections are in reasonable agreement with experiments and V-A theory. There are some remaining questions on the model in regard to $\mathrm{K}_{\mathbf{L}} \rightarrow \mu^{+} \mu^{-}, \mathrm{K}_{1}-\mathrm{K}_{2}$ mass difference; $\mathrm{I}=1 / 2$ rule, strong interaction, and $\mathrm{CP}$ nonconservation.

Pais discussed the following equalities as tests of $\mathrm{CP}$ violation in decays of charmed particle $\mathrm{D}^{+} \mathrm{D}^{-} \mathrm{D}^{\circ}$ and $\overline{\mathrm{D}}^{\circ}$ (Pais and Treiman)

$$
\begin{aligned}
& \Gamma\left(D^{+} \rightarrow \pi^{+} \pi^{0}\right)=\Gamma\left(D^{-} \rightarrow \pi^{-} \pi^{0}\right) \\
& \Gamma\left(D^{+} \rightarrow l^{+} v^{\cdots}\right)=\Gamma\left(D^{-} \rightarrow l^{-} l^{-} \cdots\right)
\end{aligned}
$$

and branching ratios of $D^{\circ}$ and $\bar{D}^{\circ}$

$$
B\left(l^{+} l^{\prime} \bar{f}\right)=\bar{B}\left(l^{-} \nu, f\right)
$$

where $f$ are hadrons and $\nu$ is neutrino or antineutrino. 
Express $D_{1}$ and $D_{2}$ in terms of $D^{\circ}$ and $\bar{D}^{\circ}$ as for $K_{S}$. and $K_{2}$, and suppose $D_{1}$ and $D_{2}$ have $C P$ impurities and $A S / \Delta C=1,0-1$ are all $C P$ conserving. Then the impurities can be due to a second superweak interaction a la L. Wolfenstein or would be due to a clash between $\Delta S / \Delta C=1$ and $\Delta S / \Delta C=-1$ interactions. The observational difference between these two cases are discussed.

Sachs reviewed the basic relations for $\mathrm{CP}$ violation in $\mathrm{K}$ decay and presented a new mechanism for $\mathrm{CP}$ violation assuming that there is no superweak interactions. The question is why are $\eta=A\left(K_{L} \rightarrow 2 \pi\right) / A\left(K_{S} \rightarrow 2 \pi\right)$ and the complex parametert that measures impurity of the $K_{\mathbf{2}} \mathrm{K}_{\mathrm{S}}$ states so small, i.e. $|n|=|t| \div 2 \times 10^{-3}$ and how can this small number arise naturally. If $\mathrm{CP}$ violation does not take place on the mass shell in the $\mathrm{K}^{\circ} \mathrm{K}^{\circ}$ decay modes, then the amplitude satisfies $\bar{A}_{\bar{c}}=A_{c}$ for a.ll channels $c$. The proposed mechanism is to assume $A_{C}(E)=\bar{A}_{C}(E)$ for large values of E) $m$ : Experimental tests for elucidating the origin of $\mathrm{T}$ violation at high energies are discussed.

Marx reviewed the vacuum degeneracy of the self coupled real scalar field and the physical consequences (for cosmology and high energy physics) of finite regions with different vacuum expectation values $\langle\phi\rangle$. Domokos et al. found that the effective weak interaction constant satisfies the relations $G_{w}=\langle\phi\rangle^{-2}$ and $\dot{G}_{w} / G_{w}=-3 H=-10^{-10}$ years ${ }^{-i}$

where $\mathrm{H}$ is the Hubble constant. When there are two vacuum states, the upper bound of the cross section for vacuum bubble production in the lower vacuum was found by Kobsarev et al. to be

$$
6(p p \rightarrow b u b b l e)<10^{-77} \mathrm{~cm}^{2}
$$

In the Kuti et al. extended particle model with quark confinement, their potential is approximated by $V(r) \cdots \frac{1}{r}+r$ 
Pais proved the following theorem regarding the number of massless particles $n$ (pseudo Goldstone bosons) which are massless in the tree approximation but may get masses in higher order (Georgi and Pais)

$$
n=D\left(S_{\lambda}\right)-D(G)+D\left(G_{\lambda}\right)
$$

where $D(G), D\left(G_{\lambda}\right)$ and $D\left(S_{\lambda}\right)$ are the dimensions of the gauge group $G$, the group after spontaneous symmetry breakdown $G_{\lambda}$ and $S_{\lambda}$ the tangent space to the surface $S$ (defined by the derivative with respect to fields $\phi_{i}$ of the potential in the tree approximation). Various examples were given of this theorem.

Kummer reviewed a new symmetry between fermion and bosons called supersymmetry (SUSY). The nice aspects are that when SUSY is spontaneously broken, one obtains a Goldstone fermion which can be identified as a neutrino and that there are fewer renormalization constants, and even with Higgs scalars the theory remains asymptotically free. The difficulties are that spontaneous breaking of SUSY is not easy, there are two neutrinos so one is still unexplained, marriage of parity conservation and spontaneous breaking is difficult, and there is a low energy theorem for $\nu$ (deWit and Freedman) which casts doubt on the Goldstone fermion idea of the $V$ in the limit the neutrino energy goes to zero.

Pietschmann gave an interesting and humorous summary of the Workshop and Stremnitzer gave a captivating sumnary of the discussion sessions. 
The Workshop was open to all interested physicists so that it offered an excellent opportunity for the participants to learn from the lectures given by the leading scholars on weak interactions and from each other in the discussion sessions. There were also ample opportunities to establish personal contacts. From the various remarks we have received. it appears that all the participants benefited from the Workshop and that such workshops should be continued to further international scien tific exchange. Some suggestions for improvement are to relate the discussion sections more closely to the lectures and to include more sessions where future experiments are intensely discussed.

It is expected that several scientific papers will result from the Workshop and be published in the scientific journals. At present, the following papers are known to us

R.N. Mohopatra - Unified Gauge Theories with Right-handed currents and Heavy Fermions.

G.J. Aubrecht, E. Takasugi and K. Tanaka - Phenomenological Bounds in Inclusive Neutrino Interactions.

The success of the Workshop is due to the combined efforts of many people. We express our gratitude for the advice and support of E.L. Jossem, the sponsors, members of the international advisory comitcee, all members of the local committee, in particular, W.F. Palmer and S.S. Pinsky for assistance in arrangements for the Workshop, and to the Workshop secretary, A. Danford, for her unselfish devotion: 